\title{
Influencia de los iones nitrato en la corrosión de la Aleoción 22
}

\author{
Nitrate lons' Influence on the Alloy 22 Corrosion Behavior \\ Influência dos íons nitrato na corrosão da Liga 22
}

Fecha recepción: 13 de julio de 2015

Fecha aprobación: 25 de agosto de 2015
Mauricio Rincón-Ortiz*

Martín Alejandro Rodríguez**

Ricardo Mario Carranza***

\section{Resumen}

La aleación 22 (N06022) pertenece a la familia de aleaciones Ni-Cr-Mo, su base es el níquel y tiene un alto contenido de cromo $(\sim 22 \%)$ y molibdeno $(\sim 13 \%)$. Diversos estudios muestran que la aleación 22 es resistente a la corrosión generalizada y localizada (en rendijas) en soluciones acuosas, con altas concentraciones de cloruro y un amplio rango de temperaturas.

Para este trabajo se aplicaron técnicas electroquímicas, tales como Espectroscopia de Impedancia Electroquímica (EIS) y ensayos Potenciodinámicos-Galvanostáticos-Potenciodinámicos (PD-GS-PD), además de técnicas complementarias no electroquímicas, como Microscopia Electrónica de Barrido, para determinar el efecto de los iones nitrato y cloruro sobre la corrosión generalizada y localizada de la aleación 22 a $90{ }^{\circ} \mathrm{C}$.

Los resultados muestran que para la relación molar crítica $\mathrm{R}=\left[\mathrm{NO}_{3}^{-}\right] /\left[\mathrm{Cl}^{-}\right] \geq 0.2$ se elimina completamente la susceptibilidad de la aleación 22 a la corrosión en rendijas. Las velocidades de corrosión obtenidas fueron del orden de $0.1 \mu \mathrm{m} / \mathrm{año}$ a 24 horas de inmersión.

Palabras clave: Aleación 22, Corrosión en rendijas, Nitratos, Potencial de repasivación, PD-GS-PD.

\section{Abstract}

Alloy 22 (N06022), is a nickel based alloy, with a high content of chromium ( 22\%) and molybdenum ( 13\%) that belongs to the Ni-Cr-Mo alloys family. Different studies show that the alloy 22 is resistant to generalized

\footnotetext{
* Ph.D. Universidad Industrial de Santander (Bucaramanga-Santander, Colombia). mrincono@saber.uis.edu.co.

** Ph.D. Instituto Sábato - Comisión Nacional de Energía Atómica (Buenos Aires, Argentina). maalrodr@cnea.gov.ar

*** Ph.D. Instituto Sábato - Comisión Nacional de Energia Atómica (Buenos Aires, Argentina). carranza@cnea.gov.ar
} 
and localized (in crevices) corrosion in aqueous solution, with high chloride concentration, and in a wide range of temperatures.

Electrochemical techniques such as Electrochemical Impedance Spectroscopy (EIS) and PotentiodynamicGalvanostatic-Potentiodynamic (PD-GS-PD) and non-electrochemical techniques such as the Scanning Electron Microscopy (SEM), were applied to determine the effect of the ionic nitrates and chlorides upon the alloy 22 at $90^{\circ} \mathrm{C}$ generalized and localized corrosion.

The results show that for a critical molar concentration ratio $\mathrm{R}=\left[\mathrm{NO}_{3}^{-}\right] /\left[\mathrm{Cl}^{-}\right] \geq 0.2$ it is completely eliminated the alloy 22 susceptibility to the crevice corrosion. The corrosion speed rates obtained were about $0.1 \mu \mathrm{m} / \mathrm{year}$ at 24 hours of immersion.

Key words: Alloy 22, Crevice corrosion, Nitrates, Repassivation potential, PD-GS-PD.

\section{Resumo}

Aliga 22 (N06022) pertence à família de ligasNi-Cr-Mo, sua base é o níquel etem um alto conteúdo de cromo $(\sim 22 \%)$ emolibdênio $(\sim 13 \%)$. Diversos estudos mostram que a liga 22 é resistente à corrosão generalizada e localizada (emfendas) em soluções aquosas, com altas concentrações de cloreto e um amplo intervalo de temperaturas.

Para este trabalho se aplicaram técnicas eletroquímicas, tais como Espectroscopia de Impedância Eletroquímica (EIS) e ensaios Potenciodinâmicos-Galvanostáticos-Potenciodinâmicos (PD-GS-PD), além de técnicas complementárias não eletroquímicas, como Microscopia Eletrônica de Varredura, para determinar o efeito dos íns nitrato e cloreto sobre a corrosão generalizada e localizada da liga 22 a $90{ }^{\circ} \mathrm{C}$.

Os resultados mostram que para a relação molar crítica $\mathrm{R}=\left[\mathrm{NO}_{3}^{-}\right] /\left[\mathrm{Cl}^{-}\right] \geq 0.2$ se elimina completamente a susceptibilidade da liga 22 à corrosão em fendas. As velocidades de corrosão obtidas foram da ordem de 0.1 $\mu \mathrm{m} /$ ano a 24 horas de imersão.

Palavras chave: Liga 22, Corrosão em fendas, Nitratos, Potencial de repassivação, PD-GS-PD.

Cómo citar este artículo:

[1] M. Rincón-Ortiz, M. A. Rodríguez \& R. M. Carranza, "Influencia de los iones nitrato en la corrosión de la Aleación 22”, Fac. Ing., vol. 24 (40), pp. 107-116, Sep.-Dic. 2015. 


\section{INTRODUCCIÓN}

El níquel puede alearse con diferentes metales para producir diversas familias de aleaciones; una de ellas es la familia Ni-Cr-Mo. La aleación 22 (Ni-22\%Cr$13 \% \mathrm{Mo}-3 \% \mathrm{~W}-3 \% \mathrm{Fe}$ ) ha sido diseñada para resistir la corrosión en condiciones tanto oxidantes como reductoras, en los medios más comunes de la industria. Gracias a sus excelentes propiedades frente a la corrosión, esta aleación es una de las candidatas para la fabricación de contenedores de residuos nucleares de nivel alto [1-5]. Los residuos nucleares de nivel alto contienen isótopos radiactivos con períodos de semidesintegración superiores a 30 años; estos residuos son emisores de calor y pueden permanecer activos durante miles o decenas de miles de años, por ello requieren sistemas de gestión definitiva que aseguren su aislamiento y confinamiento [6].

El almacenamiento geológico profundo en formaciones geológicas estables es la alternativa más firme en el ámbito mundial para la disposición final de residuos nucleares de nivel alto [6-10]. Los repositorios geológicos se basan en el principio multibarrera, que consiste en interponer una serie de barreras, naturales e ingenieriles, entre los residuos y la biosfera $[6,8]$. Las barreras naturales deben contribuir al aislamiento de los residuos, minimizando la cantidad de agua entrante al depósito y limitando el transporte de los residuos a través del sistema natural; para ello, el depósito debe estar ubicado por encima y por debajo de formaciones geológicas muy estables. Las barreras ingenieriles son diseñadas específicamente para prolongar el aislamiento de los residuos y limitar su potencial de liberación [8]; la principal barrera ingenieril es el contenedor de residuos.

Dado que los contenedores prestarán servicio en ambientes naturales caracterizados por soluciones acuosas multiiónicas [6,10], se estima que este material podría sufrir tres tipos diferentes de deterioro: corrosión general, corrosión localizada (específicamente en rendijas) y corrosión bajo tensión [10]. Desde el punto de vista de la corrosión rendijas, el ion cloruro es el principal ion agresivo [11-13]; otras especies, como nitratos, sulfatos, carbonatos, fluoruros, ácidos orgánicos, etc., pueden mitigar o inhibir la corrosión en rendijas, siempre y cuando su concentración sea lo suficientemente alta $[12,13$, 14-19]. Hay una relación crítica de concentración $\mathrm{R}_{\mathrm{CRIT}}=[$ Inhibidor $] /\left[\mathrm{Cl}^{-}\right]$para que la inhibición sea completa. Mientras más bajo sea el valor de $\mathrm{R}_{\text {CRIT }}$, más efectivo es el inhibidor. El pH de la solución no tiene un efecto importante en la iniciación y repasivación de la corrosión en rendijas, a menos que se consideren valores extremos $[19,20]$.

La corrosión generalizada y localizada (corrosión en rendijas) ha sido y es extensivamente estudiada en presencia de soluciones que simulan los componentes de aguas subterráneas $[10,17,21]$. Se encontró, en general, que la aleación es muy resistente a estos tipos de corrosión en los probables medios de un repositorio.

El objetivo de este trabajo fue evaluar el comportamiento frente a la corrosión general y localizada (corrosión en rendijas) de la aleación 22 en soluciones que contienen diferentes concentraciones de cloruros con adiciones de nitratos, utilizando métodos y parámetros electroquímicos.

\section{Procedimiento experimental}

Las mediciones electroquímicas se llevaron a cabo utilizando una celda de vidrio. Se utilizó como contraelectrodo una lámina de platino $\mathrm{y}$ un electrodo de referencia de calomel saturado (ECS) $\left(\mathrm{V}_{\mathrm{ECS}}=\mathrm{V}_{\mathrm{ENH}}+0,244 \mathrm{~V}\right)$, el cual se conectó con la solución mediante un capilar de Luggin. La celda se mantuvo a una temperatura constante de $90{ }^{\circ} \mathrm{C}$ mediante un baño de termostático de agua. Los estudios se efectuaron utilizando dos tipos de probetas, geométricamente diferentes, de aleación 22.

1) Probetas PCA (Prism Crevice Assembly) con formadores de rendijas de material cerámico recubierto con PTFE(ASTM G78) [22], especialmente diseñadas para el estudio de la corrosión en rendijas, con dimensiones aproximadas de $19 \mathrm{~mm}$ x $19 \mathrm{~mm}$ x 9,5 mm y un área expuesta de, aproximadamente, $14 \mathrm{~cm}^{2}$.

2) Probetas prismáticas para el estudio de la corrosión en estado activo y pasivo, las cuales presentan unas dimensiones aproximadas de $12 \mathrm{~mm}$ x $12 \mathrm{~mm} \times 15 \mathrm{~mm}$ y un área expuesta a la solución de, aproximadamente, $10 \mathrm{~cm}^{2}$.

Las muestras tuvieron un acabado superficial mediante pulido con papel de lija de carburo de silicio número 600; se les removió la grasa con alcohol, se lavaron 
con agua destilada y se les aplicó un torque de 5 N.m. El pulido se realizó 1 hora antes del ensayo.

Se determinó la susceptibilidad a la corrosión en rendijas mediante la realización de curvas de polarización por la técnica PotenciodinámicaGalvanostática-Potenciodinámica

(PD-GS-PD) propuesta por Mishra y Frankel [23]. Se utilizó una velocidad de barrido de $0,167 \mathrm{mV} / \mathrm{s}$. La técnica (PD-GS-PD) consta de tres etapas: 1) una polarización potenciodinámica $(\mathrm{a} 0,167 \mathrm{mV} / \mathrm{s})$ en la dirección anódica hasta alcanzar una corriente anódica de $30 \mu \mathrm{A}$; 2) la aplicación de una corriente anódica constante de $\mathrm{I}_{\mathrm{GS}}=30 \mu \mathrm{A}\left(\mathrm{I}_{\mathrm{GS}}=2 \mu \mathrm{A} / \mathrm{cm}^{2}\right.$, aproximadamente) por 2 horas, y 3 ) una polarización potenciodinámica (a $0,167 \mathrm{mV} / \mathrm{s}$ ) en la dirección catódica, desde el potencial anterior hasta alcanzar la repasivación de la aleación.

El potencial de repasivación de la corrosión en rendijas para esta técnica es definido en la intersección de dos barridos, el de la etapa 1 y el de la etapa 3. Se utilizaron soluciones acuosas de $\mathrm{NaCl}$ $1 \mathrm{M}, 0,1 \mathrm{M}$, con diferentes concentraciones de nitratos $\mathrm{NaNO}_{3}$, desaireadas con burbujeo de nitrógeno y a una temperatura de $90{ }^{\circ} \mathrm{C}$. El cociente entre las concentraciones de nitratos y de cloruros varió entre 0.01 y $0.5\left(\mathrm{R}=\left[\mathrm{NO}_{3}^{-}\right] /\left[\mathrm{Cl}^{-}\right]\right)$. Se registró la evolución del potencial de corrosión durante 24 horas en soluciones de cloruro con adiciones de nitrato.

Se obtuvo la velocidad de corrosión generalizada mediante Espectroscopía de Impedancia Electroquímica (EIS). Se realizaron ensayos al potencial de corrosión luego de $1 \mathrm{~h} \mathrm{y} 24 \mathrm{~h}$ de inmersión. Se aplicó una señal sinusoidal de potencial, realizándose un barrido de frecuencias entre $10 \mathrm{kHz}$ y $1 \mathrm{mHz}$. Circuitos equivalentes sencillos se ajustaron a los datos experimentales con el fin de obtener la resistencia de polarización $\left(\mathrm{R}_{\mathrm{P}}\right) \mathrm{y}$ calcular la velocidad de corrosión $\left(\mathrm{V}_{\mathrm{CORR}}\right),(1)$ :

$$
\mathrm{V}_{\text {corr }}(\mu \mathrm{m} / \text { año })=\frac{K \text { B PE }}{\rho} \frac{1}{\mathrm{R}_{\mathrm{P}}}
$$

En donde $\mathrm{K}$ es la constante de conversión faradaica $\left(\mathrm{K}=3,2710^{6} \mu \mathrm{m} \mathrm{A}^{-1} \mathrm{~cm}^{-1} \mathrm{año}^{-1}\right)$, B es la constante de Stern y Geary, $\mathrm{PE}$ es el peso equivalente $(\mathrm{PE}=23,28)$, $\rho$ es la densidad de la aleación $\left(\rho=8,69 \mathrm{~g} / \mathrm{cm}^{3}\right)$ y $\mathrm{R}_{\mathrm{p}}$ es la resistencia de polarización. Al finalizar los ensayos se observaron las probetas en el microscopio óptico y en el Microscopio Electrónico de Barrido.

\section{Resultados}

En las Figs. 1 y 2 se muestran las curvas (PD-GS-PD) obtenidas para corrosión en rendijas, en soluciones de cloruros $0,1 \mathrm{M}$ y $1 \mathrm{M}$ con diferentes adiciones de nitratos. En todos los casos se observó una región catódica inicial a potenciales más negativos que $-0,5 \mathrm{~V}$, una región de pasividad donde la densidad de corriente se mantuvo relativamente constante y baja (alrededor de $1 \mu \mathrm{A} / \mathrm{cm}^{2}$ ) y, por último, una zona transpasiva en la que al incrementar el potencial aplicado, la densidad de corriente anódica se incrementó rápidamente. El rango de potencial dentro del cual se observó pasividad resultó menor en soluciones con menor concentración de nitratos, y viceversa, mayor en aquellas soluciones con mayor concentración.

Se observó una histéresis entre los barridos potenciodinámicos en sentido catódico luego de la aplicación de una corriente anódica constante de $\mathrm{I}_{\mathrm{GS}}=30 \mu \mathrm{A}$, y los barridos potenciodinámicos en el sentido anódico de las curvas realizadas en soluciones de cloruro $0,1 \mathrm{M}$ y $1 \mathrm{M}$ con diferentes concentraciones de nitratos. En soluciones con una mayor concentración de nitratos disminuyó en una forma muy apreciable dicha histéresis, independientemente de la concentración de cloruros. Se observaron diferencias en la susceptibilidad a la corrosión en rendijas atribuibles a la presencia de nitratos. No se observó una variación del pH de las soluciones antes y después de cada experiencia. 


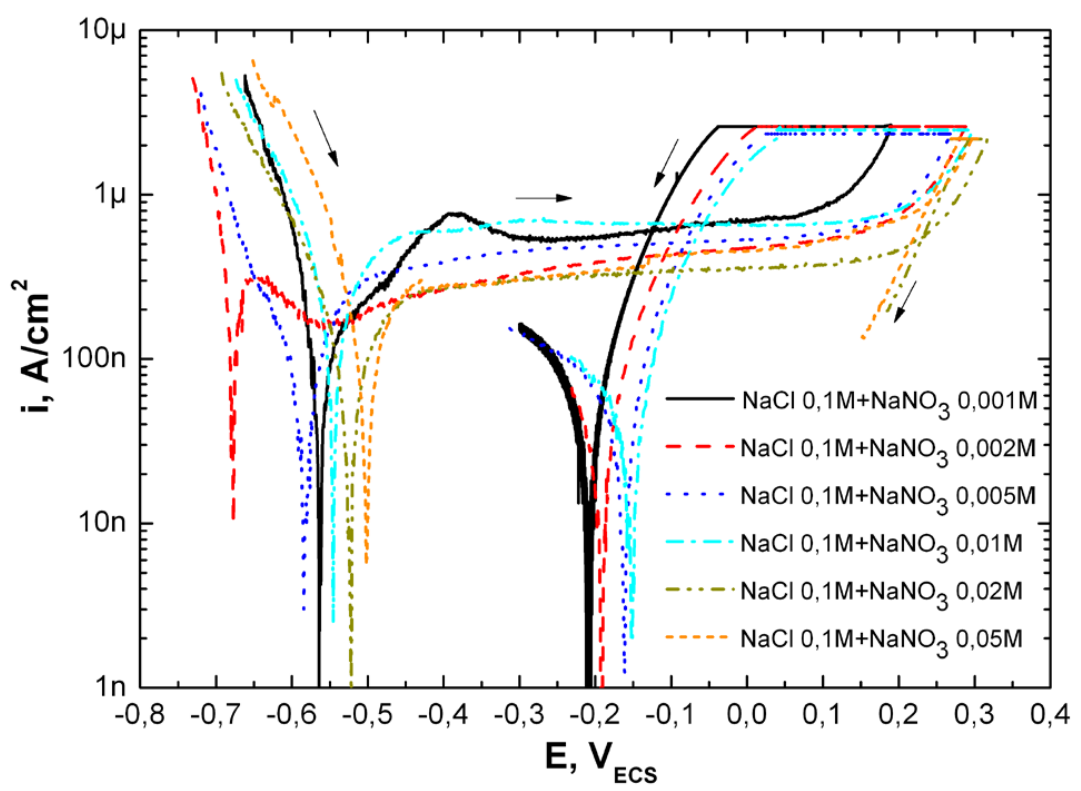

Fig. 1. Curvas de PD-GS-PD de la aleación 22 en soluciones acuosas desaireadas de $\mathrm{NaCl} 0.1 \mathrm{M}$ con diferentes adiciones de nitratos a $90^{\circ} \mathrm{C}$

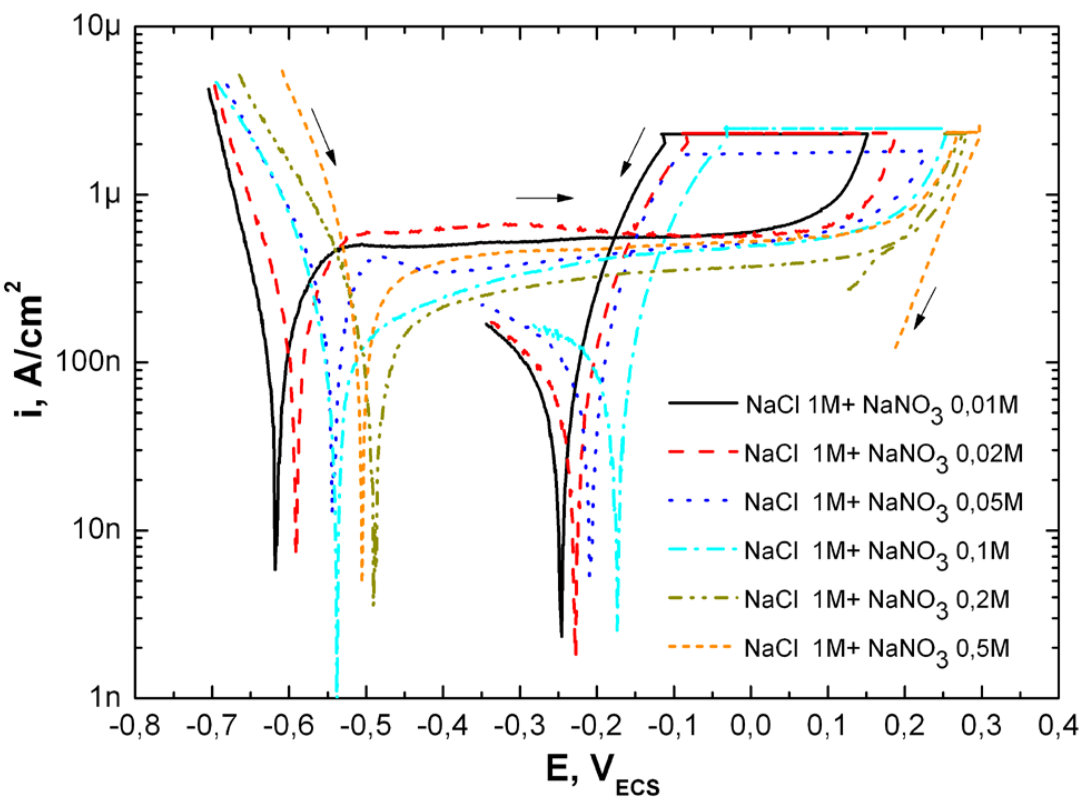

Fig. 2. Curvas de PD-GS-PD de la aleación 22 en soluciones acuosas desaireadas de $\mathrm{NaCl} 1 \mathrm{M}$ con diferentes adiciones de nitratos a $90^{\circ} \mathrm{C}$

Uno de los potenciales críticos de la corrosión en rendijas es el de repasivación $\left(\mathrm{E}_{\mathrm{CO}}\right)$. Se definió el $\mathrm{E}_{\mathrm{CO}}$ como la intersección de dos barridos, de la etapa 1 y la etapa 3, según el método PD-GS-PD. En la Fig. 3 se muestran los potenciales de repasivación para soluciones con concentraciones $0,1 \mathrm{M}$ y $1 \mathrm{M}$ de cloruros, con adiciones de diferentes concentraciones de nitratos. Se observó un aumento considerable en el potencial de repasivación para una relación molar de $0.2(\mathrm{R}=0.2)$. 


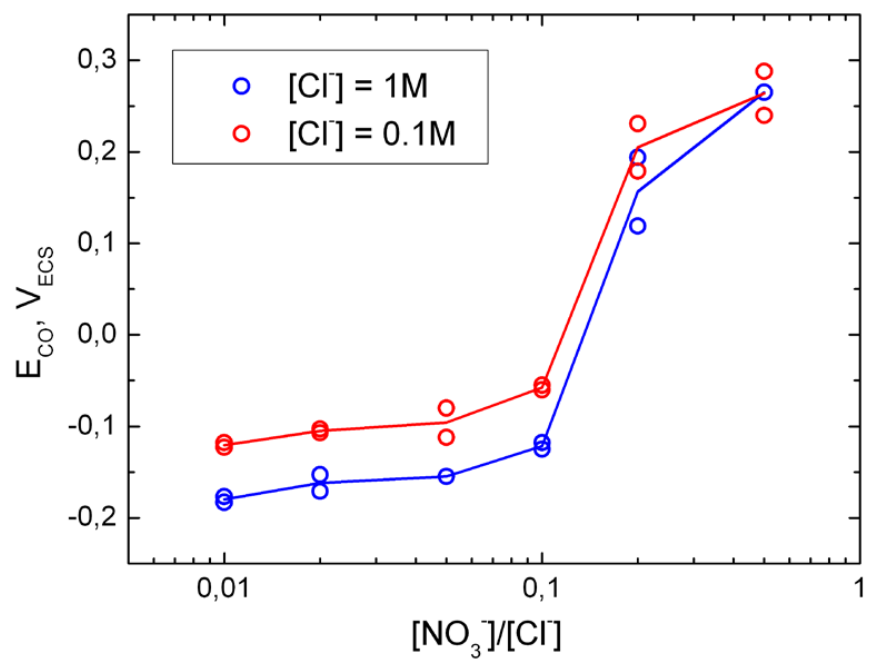

Fig. 3. Efecto de R= [NO3- $] /[\mathrm{Cl}-]$ sobre el potencial de repasivación ECO para la aleación 22 a $90{ }^{\circ} \mathrm{C}$

En la Fig. 4 se aprecia la forma del ataque por corrosión en rendijas, el cual siempre se presentó debajo de los formadores de rendijas. El ataque fue disminuyendo a medida que aumentó la concentración de nitratos. Para un $\mathrm{R}=0.2$ se observa la inhibición total de la corrosión en rendijas de la aleación 22.
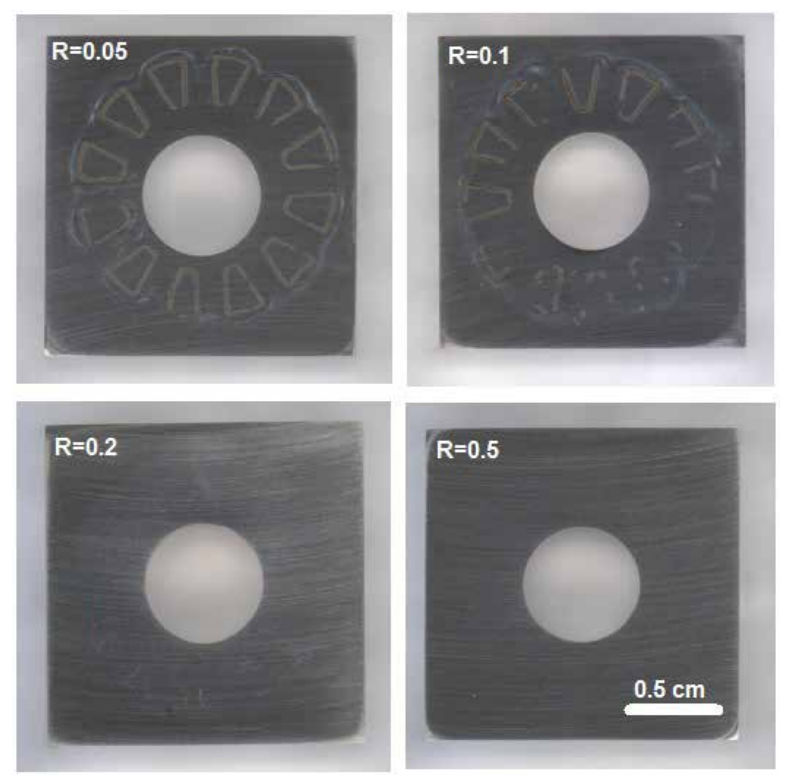

Fig. 4. Fotografías de probetas PCA, luego de una curva PD-GS-PD, donde se muestra el efecto de R=[NO3-]/[Cl-]

En la Fig. 5 se observa en detalle, por medio de la técnica de Microscopía Electrónica de Barrido (SEM, por su nombre en inglés), una probeta expuesta a una solución $\mathrm{NaCl} 1 \mathrm{M}-\mathrm{NaNO}_{3}$ 0,05 M. Se encontró un revelado de granos en algunas zonas donde el ataque fue menor, además de productos de corrosión adyacentes a las áreas atacadas por corrosión en rendijas. 

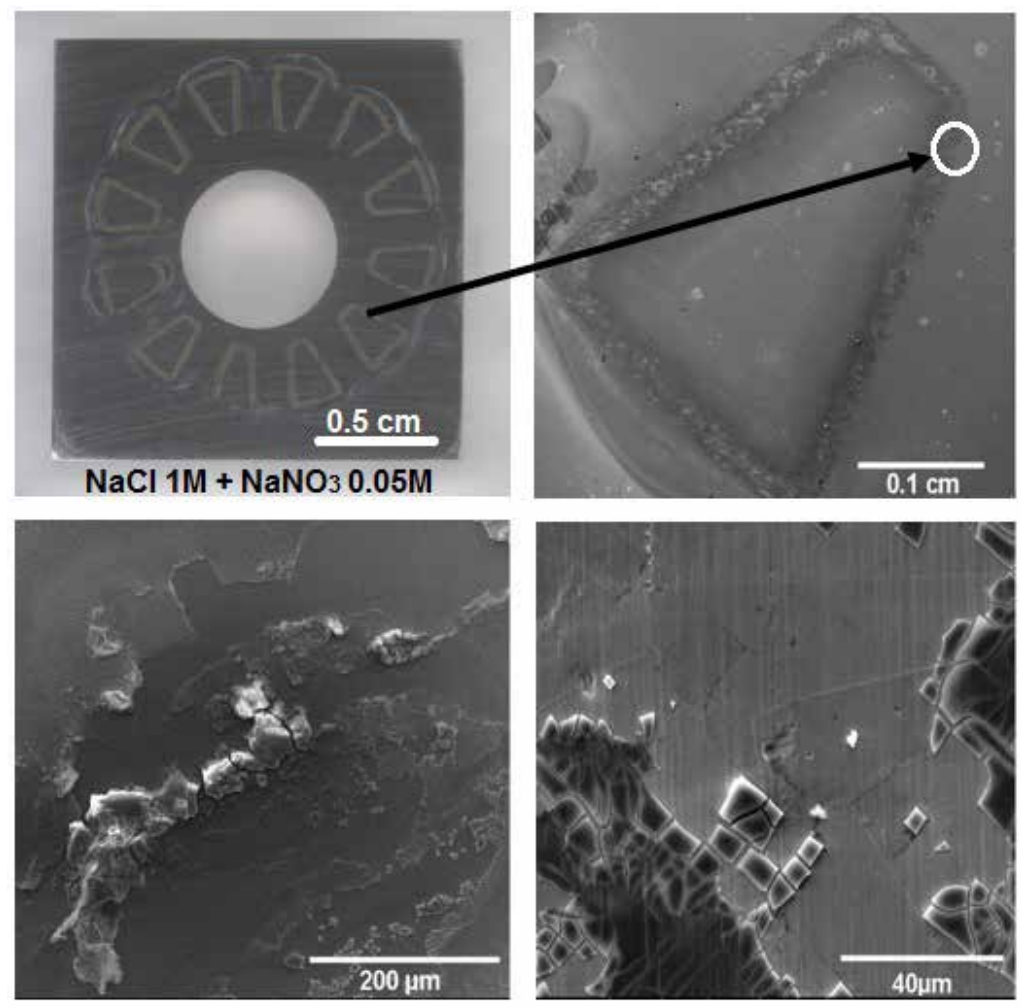

Fig. 5. Imagen SEM de la aleación 22 luego de un ensayo PD-GS-PD en solución desaireada $\mathrm{NaCl} 1 \mathrm{M}+\mathrm{NaNO} 30.05$ M. $\mathrm{R}=0,05$.

En la Fig. 6 se encontró que la probeta no presentó ningún ataque localizado cuando fue expuesta a una solución $\mathrm{NaCl} 1 \mathrm{M}-\mathrm{NaNO}_{3}$ 0,2 M. En la imagen de SEM se pueden distinguir las superficies debajo de los dientes de los formadores de rendijas debido a que allí no se formó la película de óxidos de la transpasividad al no estar en contacto con la solución.

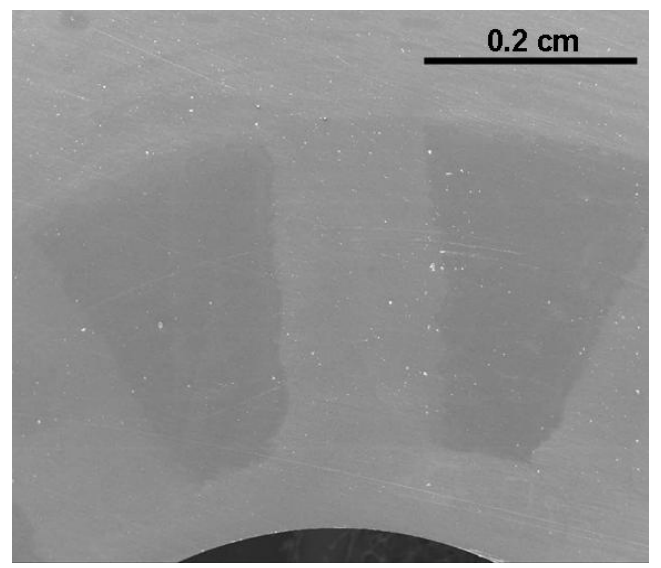

Fig. 6. Imagen SEM de la aleación 22 luego de un ensayo PD-GS-PD en solución desaireada $\mathrm{NaCl} 1$ $\mathrm{M}+\mathrm{NaNO} 30.2 \mathrm{M} . \mathrm{R}=0,2$
La Fig. 7 muestra el diagrama de Bode obtenido en la medida de EIS de una solución $\mathrm{NaCl} 1 \mathrm{M}+\mathrm{NaNO}_{3}$ $0.5 \mathrm{M}$. En todas las soluciones estudiadas se obtuvieron diagramas puramente capacitivos, para los cuales un circuito equivalente $\mathrm{R}_{\Omega^{-}}\left(\mathrm{R}_{\mathrm{p}} / \mathrm{CPE}\right) \mathrm{se}$ ajustó correctamente a los resultados experimentales. En la Fig. 8 se presentan las velocidades de corrosión en soluciones aireadas naturalmente, calculadas con el parámetro $\mathrm{R}_{\mathrm{p}}$, obtenido por medio del ajuste de circuitos equivalentes a los datos de EIS. Teniendo en cuenta las mediciones realizadas después de 1 hora de inmersión, hubo una disminución de la velocidad de corrosión en las soluciones que contenían una menor concentración de cloruro y nitrato para una misma relación molar $\left(\mathrm{R}=\left[\mathrm{NO}_{3}^{-}\right] /\left[\mathrm{Cl}^{-}\right]\right)$. Sin embargo, después de 24 horas de inmersión la velocidad de corrosión fue aproximadamente de $0,1 \mu \mathrm{m} / \mathrm{año}$ e independiente de la concentración de nitrato. 


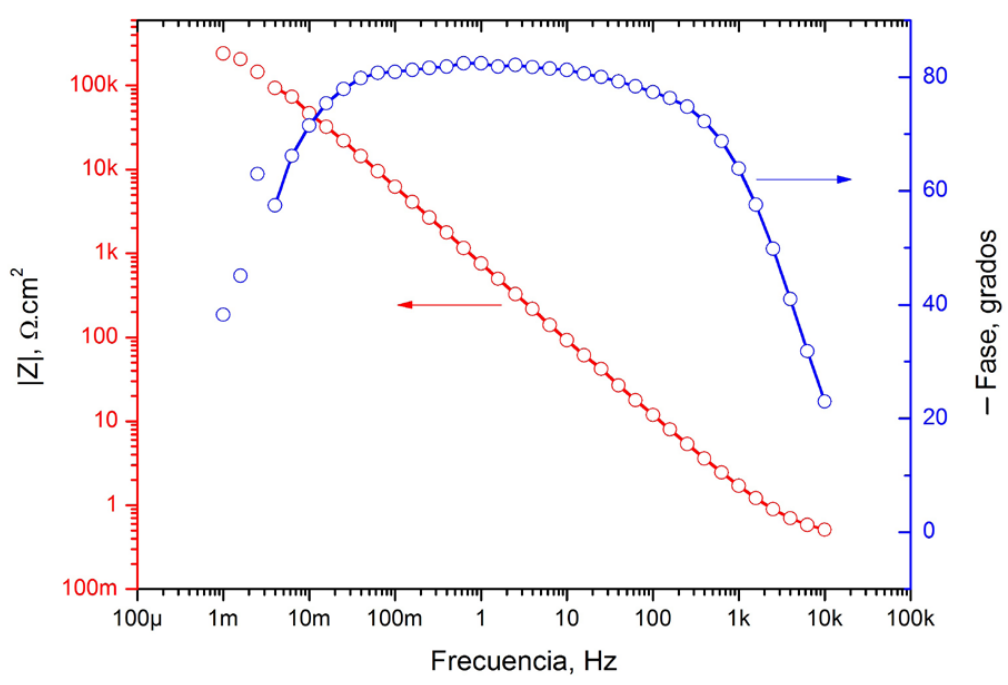

Fig. 7. Diagrama de Bode obtenido al potencial de corrosión a 24 h de inmersión en solución de $\mathrm{NaCl} 1 \mathrm{M}+\mathrm{NaNO} 3$ 0.5

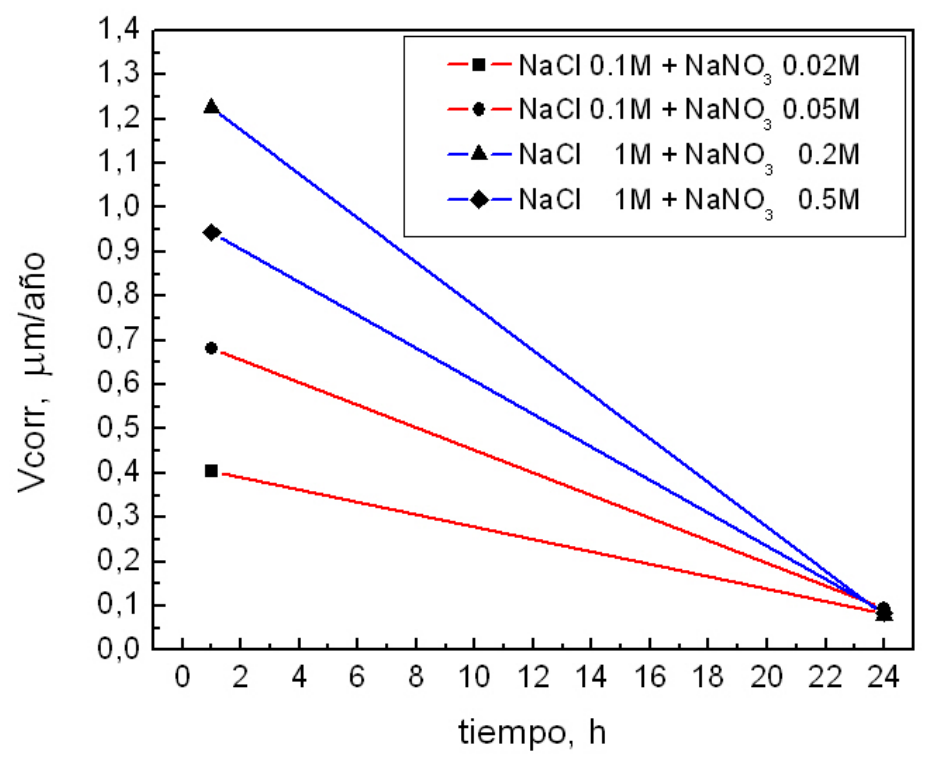

Fig. 8. Velocidad de corrosión en función del tiempo de inmersión para soluciones de $\mathrm{NaCl} 0,1 \mathrm{M}$ y $1 \mathrm{M}$ con diferentes concentraciones de nitratos, en medios aireados, a $90{ }^{\circ} \mathrm{C}$

\section{Discusión}

Diversas investigaciones se han realizado recientemente para establecer la susceptibilidad de la aleación 22 a la corrosión localizada en ambientes agresivos, a través de la determinación del potencial de repasivación [23]. Los ensayos electroquímicos llevados a cabo para la corrosión localizada muestran que la presencia de nitratos en la solución extiende el rango de potenciales dentro del cual la aleación 22 muestra pasividad. En todos los casos, la densidad 
de corriente de pasividad estuvo en el orden de 1 $\mu \mathrm{A} / \mathrm{cm}^{2}$ (Figs. 1 y 2.). Mediante la técnica PD-GS$\mathrm{PD}$ se determinaron las concentraciones de nitrato requeridas para la inhibición total de la corrosión en rendijas de la aleación 22 a $90{ }^{\circ} \mathrm{C}$. Se encontró que para un $\mathrm{R}=\left[\mathrm{NO}_{3}^{-}\right] /\left[\mathrm{Cl}^{-}\right] \geq 0.2$ se da la inhibición completa. Dunn y Col. [24], utilizando el método Cyclic Potentiodynamic Polarization (CPP), han reportado valores de $\mathrm{R}_{\text {CRIT }}$ de 0.12 , en los cuales se ha presentado la inhibición total de la corrosión en rendijas en soluciones de cloruros con adiciones de nitratos. En la literatura se reporta que valores de $\mathrm{R}=[$ Anión $] /\left[\mathrm{Cl}^{-}\right] \approx 0.1$ son suficientes para inhibir la corrosión en rendijas de la aleación 22 en presencia de nitratos, sulfatos y carbonatos [24]. Para iones $\mathrm{F}^{-}$ se necesitan $\mathrm{R}>2$ para inhibir totalmente la corrosión en rendijas producida en soluciones de $\mathrm{NaCl} 0.01 \mathrm{M}$ y $\mathrm{R}>7$ para $\mathrm{NaCl} 0.1$ y $1 \mathrm{M}$ [25]. Se nota una fuerte dependencia del potencial de repasivación $\mathrm{E}_{\mathrm{CO}}$ con la relación $\mathrm{R}$, independiente de $\left[\mathrm{NO}_{3}^{-}\right]$y $\left[\mathrm{Cl}^{-}\right]$, como se muestra en la Fig. 3. Es un hecho generalmente aceptado que durante la propagación de la corrosión en rendijas se forma una solución ácida dentro de la rendija [26, 27, 28]. En una solución de $\mathrm{pH}$ bajo de una rendija en proceso de corrosión, la presencia de nitrato formará ácido nítrico y se promoverá la pasivación de la aleación 22 por medio de la reposición de la capa de óxido de cromo que fue destruida por el ácido clorhídrico. De igual modo, los aniones reducibles, tales como el nitrato, inhibirán el proceso de corrosión localizada, porque al reducirse dentro de una picadura consumirán protones (2) [29].

$$
2 \mathrm{NO}_{3}^{-}+12 \mathrm{H}^{+}+10 \mathrm{e}^{-} \rightarrow \mathrm{N}_{2}+6 \mathrm{H}_{2} \mathrm{O}
$$

Estudios recientes en cuanto a la determinación de potenciales de repasivación de la aleación 22 en soluciones de cloruro a $90{ }^{\circ} \mathrm{C}$ a diferentes concentraciones señalan que la técnica PD-GS-PD presenta valores de potenciales de repasivación mucho más conservadores que los encontrados por otros métodos electroquímicos [30].

Las morfologías de ataque por corrosión en rendijas de la aleación 22 en soluciones de cloruros con nitratos son similares a las morfologías de ataque por corrosión en soluciones de cloruro encontradas en la bibliografia (Fig. 5). El tipo de ataque sufrido por medio de cloruros a la aleación 22 se puede considerar de tipo cristalino, donde los diferentes planos cristalinos se corroen a velocidades distintas [12].
Los resultados del presente trabajo, además de mostrar el buen comportamiento de los nitratos como inhibidores de la corrosión en rendijas de la aleación 22 , muestran velocidades de corrosión uniforme después de 24 horas de inmersión relativamente bajas independientes de la concentración de aniones (Fig. 8).

\section{Conclusiones}

- Se observó inhibición de la corrosión en rendijas de la aleación 22 en soluciones que contienen cloruro y nitrato a partir de una relación de concentraciones $\mathrm{R}=0.2$.

- Con la utilización de la técnica PD-GS-PD se obtuvieron valores de $\mathrm{R}_{\text {CRIT }}$ más conservadores, comparados con los obtenidos por otras técnicas electroquímicas.

- Se observaron bajas velocidades de corrosión uniforme a 24 horas de inmersión, y estas fueron independientes de la concentración de aniones.

\section{Referencias}

[1] R. B. Rebak, "Metallurgical effects on the corrosion behavior of nickel alloys", ASM Metals Handbook, Vol. 13A, Corrosion: Fundamental, Testing, and Protection, pp. 279286, ASM International, 2003.

[2] Hastelloy C-22 Alloy, Haynes International, brochure H-2019F, 2002.

[3] N. S. Zadorozne, M. C. Goirdano, R. B. Rebak et al., "Anodic Behavior of Alloy 22 in Bicarbonate Media: Effect of Alloying", Procedia Materials Science, Vol. 8, pp. 510518, 2015.

[4] A. I. Asphahani, "Advances in the development of Ni-Cr-Mo-W alloy systems whit improved resistance to aqueous corrosion", The Arabian Journal for Science and Engineering, pp. 317335, 1989.

[5] H. M. Tawancy, R.B. Herchenroeder and A.I. Asphahani, "High-performance Ni-Cr-Mo-W alloys", Journal of Metals, pp. 37-43, 1983.

[6] Geological Challenges in Radioactive Waste Isolation. Third Worldwide Review, Eds. P.A. Whiterspoon and G.S. Bodvarsson, University of California, Berkeley, CA, USA (2001). 
[7] K. D. Crowley and J. F. Ahearne, "Managing the environmental legacy of U.S. nuclear-weapons production", American Scientist, 90, 6, 514 (2002).

[8] Yucca Mountain Project, Eds. G. S. Bodvarsson, C.K. Ho, and B.A. Robinson, Elsevier (2003).

[9] Yucca Mountain Science and Engineering Report, U. S. Department of Energy, Office of Civilian Radioactive Waste Management, DOE/ RW-0539, Las Vegas, NV, USA (2001).

[10] G. M. Gordon, "F.N Speller Award Lecture: Corrosion Considerations Related to Permanent Disposal of HigH-Level Radioactive waste", Corrosion 58, 10, 811(2002).

[11] R. B. Rebak, Materials science and technology. A comprehensive treatment. Corrosion and environmental degradation, Vol. II pp. 69-111, Vol. Ed. M. Schuntze, Wiley, VCH, Weinheim, Germany (2000).

[12] R. B. Rebak, "Factors affecting the crevice corrosion susceptibility of Alloy 22", Paper $\mathrm{N}^{\circ}$ 05610, Corrosion/05, NACE Intl., Houston, TX (2005).

[13] D. S. Dunn, Y.-M. Pan, K.T. Chiang, G.A. Cragnolino and X. He, "The localized corrosion resistance and mechanical properties of alloy 22 waste package outer containers", Journal of Metals, p. 49, (2005).

[14] B. A. Kehler, G. O. Llevbare and J. R. Scully, "Crevice Corrosion Stabilization and Repassivation Behavior of Alloy 625 and Alloy 22". Corrosion, 57, 12 (2001)1042.

[15] D. S. Dunn, L. Lang, C. Wu and G.A. Cragnolino, "Effect of inhibiting oxyanions on the localized corrosion susceptibility of waste package container materials", SBNWM XXVII, MRS, Warrendale, PA, USA, Symposium Proceedings, Vol. 824, Paper CC1.7.1, 2004.

[16] G. O. Llevbare, "Effect of sulfate on the passive and crevice corrosion propierties of Alloy 22 in 4M sodium Chloride". Corrosion, 62, 4, 340 (2006).

[17] D. S. Dunn, G. A. Cragnolino, Y.-M. Pan, and L. T. Yang, Effect of Fabrication Processes on Alloy 22, Paper No. 03697, Corrosion/2003, NACE International, Houston, Texas, 2003.

[18] R. M. Carranza, Environmental and Metallurgical Variables Affecting Crevice Corrosion Susceptibility of Alloy 22, Paper
08580, Corrosion/2008, NACE Int. Houston, TX, 2008.

[19] R. M. Carranza, Journal of Metals, p. 58 (January 2008).

[20] R. M. Carranza, M. Rincón Ortiz, M. A. Rodríguez, and R. B. Rebak, Corrosion resistance of Alloy 22 in chloride and silicate solutions, 14th International Conference on Environmental Degradation of Materials in Nuclear Power Systems-Water Reactors, August 23-27, ANS, Virginia Beach, VA (2009).

[21] R. B. Rebak, T.S.E. Summers, T. Lian, R.M. Carranza, J.R. Dillman, T. Corbin, and P. Crook, Effect of thermal aging on the corrosion behavior of wrought and welded alloy 22, paper 02542, Corrosion/02 (NACE International, 2002: Houston, TX), pp. 1-17.

[22] Annual Book of ASTM Standards, G78-95, p. 315 y G61-86, p. 239, 1996.

[23] A. K. Mishra and G. S. Frankel, "Crevice corrosion repassivation of alloy 22 in aggressive environments", Corrosion Science, pp. 836844, 2008.

[24] D. S. Dunn, Y. M. Pan, L. Yang, G. A. Cragnolino, Corrosion 61, 11, p. 1.076, 2005.

[25 ] R. M Carranza, M. A Rodríguez and R. B Rebak, "Effect of Fluoride Ions on Crevice Corrosion and Passive Behavior of Alloy 22 in Hot Chloride Solutions", Corrosion 63, 5, p. 480-490, 2007.

[26] J. R. Galvele, "Transport processes and the mechanism of pitting of metals", Journal of the Electrochemical Society, 123, 4, p. 464, 1976.

[27] Z. Szklarska-Smialowska, Pitting and crevice corrosion, NACE Int., Houston, TX, USA, 2005.

[28] P. Combrade, Crevice corrosion of metallic materials in corrosion mechanisms in theory and practice, second edition, ed. P. Marcus, Marcel Dekker, 2000.

[29] J. R. Galvele y G. S. Duffó, Degradación de Materiales -I. Corrosión, Buenos Aires: Jorge Baudino Ediciones, Instituto Sabato, UNSAMCNEA, 2006.

[30] M. Rincón Ortiz, M. A. Rodríguez, R. M Carranza, R. B Rebak. Crevice corrosion repassivation of alloy 22 in chloride solutions, NACE 2010, paper 10237. 